\title{
Connectivity Preserving Distributed Swarm Aggregation for Multiple Kinematic Agents
}

\author{
Dimos V. Dimarogonas and Kostas J. Kyriakopoulos
}

\begin{abstract}
A distributed swarm aggregation algorithm is developed for a team of multiple kinematic agents. Each agent is assigned with a control law which is the sum of two elements: a repulsive potential field, which is responsible for the collision avoidance objective, and an attractive potential field, that drives the agents to a configuration where they are close to each other and forces the agents that are initially located within the sensing radius of an agent to remain within this area for all time. In this way, the connectivity properties of the initially formed communication graph are rendered invariant for the trajectories of the closed loop system. It is shown that agents converge to a configuration where each agent is located at a bounded distance from each of its neighbors. The results are also extended to the case of nonholonomic kinematic agents and to the dynamic edge addition case, for which we derive a better bound in the swarm size than in the static case.
\end{abstract}

\section{INTRODUCTION}

Distributed multi-agent control is a field that has recently gained increasing attention, due to the need for autonomous control of more than one mobile agent in the same workspace. The main variations of the approaches so far lie in the specifications that the control design should impose on the multi-agent team. These among others include formation stabilization [1],[2], flocking behavior [4], [12],[8] and swarm navigation with collision avoidance [2], [3].

The objective of this paper is distributed swarm aggregation with collision avoidance. Each agent is assigned with a control which is the sum of two elements: a repulsive potential, responsible for the collision avoidance objective, and an attractive potential, that drives the agents close to each other and also forces the agents that are initially located within the sensing zone of an agent to remain within this zone for all time. Hence the control design renders the set of edges of the initially formed communication graph positively invariant for the trajectories of the closed loop system. In this way, if the communication graph, which is formed based on the initial displacement between the agents, is connected, then it remains connected for all time. Connectivity preserving algorithms for multi-agent systems with linear motion models were recently presented in [5],[7].

This model was also used in [3] where the authors used a potential field, consisting of the sum of a repulsive and an attractive term. The innovation of our approach is the fact

Dimos Dimarogonas is with the Automatic Control Lab., School of Electrical Engineering, Royal Institute of Technology,Stockholm, Sweden \{dimosdee.kth.se\}. Kostas Kyriakopoulos is with the Control Systems Lab, Department of Mechanical Engineering, National Technical University of Athens, Greece $\{k$ kyria@mail.ntua.gr $\}$. This work was supported by the EU through contract I-SWARM (IST-2004-507006). that the control design is distributed. The collision avoidance objective is guaranteed through the use of repulsive potentials that disappear whenever agents are outside the sensing zone of one another, respecting the agents' limited sensing capabilities. We also provide a control law that renders the connectivity properties of the initially formed communication graph invariant for the trajectories of the closed loop system, and treat the dynamic edge addition case as well. In the latter case, it is shown that the resulting swarm size is smaller than that of the static graph case treated previously. Finally, the results hold for the case of nonholonomic (unicycle type) robots as well.

\section{System AND Problem Definition}

The system consists of $N$ (point) agents operating in the same workspace $W \subset \mathbb{R}^{2}$. We denote the position of agent $i$ by $q_{i} \in W$. The configuration space is spanned by $q=$ $\left[q_{1}^{T}, \ldots, q_{N}^{T}\right]^{T}$. The motion of each agent is described by:

$$
\dot{q}_{i}=u_{i}, i \in \mathcal{N}=[1, \ldots, N]
$$

where $u_{i} \in \mathbb{R}^{2}$ denotes the velocity (control input) of $i$.

For the objective of swarm aggregation, each agent $i$ is assigned to a specific subset $N_{i}$ of the rest of the team, called agent $i$ 's communication set, that includes the agents with which it can communicate in order to achieve the desired aggregation objective. Inter-agent communication can be encoded in terms of a communication graph, $G=\{V, E\}$, which is an undirected graph consisting of a set of vertices $V=\{1, \ldots, N\}$ indexed by the team members, and a set of edges, $E=\left\{(i, j) \in V \times V \mid i \in N_{j}\right\}$, containing pairs of agents that represent inter-agent communication specifications. The definition of the set $N_{i}$ is provided later.

Moreover, it is required that the agents do not collide with each other, in the sense that they are never found at the same point in the workspace. The collision avoidance procedure is distributed. Each agent has to have only local knowledge of the agents that are very close at each time instant. Since agent $i$ can sense agents located at a distance no larger than $d$, we assume that for the collision avoidance objective, agent $i$ has knowledge of the positions of agents located at a distance no larger than a radius $d_{1}$, where $0<d_{1} \leq d$, at each time instant. The subset of $\mathcal{N}$ including the agents that are located at a distance no larger than a radius $d_{1}$ from agent $i$ is denoted by $M_{i}$. Hence $M_{i}=\left\{j \in \mathcal{N}, j \neq i:\left\|q_{i}-q_{j}\right\| \leq d_{1}\right\}$. While $M_{i}$ contains the agents located at a distance no larger than $d_{1}$ from $i$ at each time instant, the communication set $N_{i}$ is defined in a slightly different manner in relation with the proposed control design. Specifically, it will be shown 
that the controller forces the agents that are initially located within the sensing zone of an agent to remain within this area for all time. In this way, no edges are lost and if the communication graph is initially connected, then it remains connected for all time. Thus, $N_{i}$ is defined as the set that agent $i$ can sense when located at its initial position, $q_{i}(0)$ :

$$
N_{i}=\left\{j \in \mathcal{N}, j \neq i:\left\|q_{i}(0)-q_{j}(0)\right\|<d\right\} .
$$

Let $G=(V, E)$ denote the initially formed communication graph under the ruling (2). Hence an edge between agents $i, j$ exists whenever they are initially located within distance $d$ from each other, i.e. $(i, j) \in E \Leftrightarrow j \in N_{i}$ if and only if $\left\|q_{i}(0)-q_{j}(0)\right\|<d$. By showing that for all pairs of agents $(i, j)$ s.t. $\left\|q_{i}(0)-q_{j}(0)\right\|<d$ the proposed controller guarantees that $\left\|q_{i}(t)-q_{j}(t)\right\|<d$ for all $t>0$, the edges are guaranteed to remain invariant (i.e. agents $i, j$ remain within distance $d$ from one another) and hence the communication graph itself, remains invariant throughout the closed loop system evolution. This result is stated and proved in Lemma 3 of the paper. The case of dynamic edge addition will be considered in Section VI. On the other hand, $M_{i}$ may change over time at time instances when an agent $j \neq i$ enters or leaves the set $\left\{q:\left\|q_{i}-q\right\| \leq d_{1}\right\}$. The (distributed) control law is of the form $u_{i}=u_{i}\left(q_{i}, q_{j}\right), j \in N_{i} \cup M_{i}$.

\section{Control Strategy}

We first define a repulsive potential field $V_{i j}: \mathbb{R}^{2} \rightarrow \mathbb{R}_{+}$to deal with the collision avoidance objective between agents $i$ and $j \in M_{i} . V_{i j}$ is required to have the following properties: (1) $V_{i j}$ is a function of the square norm of the distance of $i, j$, i.e. $V_{i j}=V_{i j}\left(\beta_{i j}\right)$, where $\beta_{i j}=\left\|q_{i}-q_{j}\right\|^{2}$, (2) $V_{i j} \rightarrow \infty$ whenever $\beta_{i j} \rightarrow 0$, (3) it is everywhere continuously differentiable, (4) $\frac{\partial V_{i j}}{\partial q_{i}}=0$ and $V_{i j}=0$ whenever $\beta_{i j}>d_{1}^{2}$, and (5) the partial derivative $\rho_{i j} \triangleq$ $\frac{\partial V_{i j}}{\partial \beta_{i j}}$ satisfies $\rho_{i j}<0$ for $0<\beta_{i j}<d_{1}^{2}$ and $\rho_{i j}=0$ for $\beta_{i j} \geq d_{1}^{2}$. It is easily seen that if $V_{i j}$ satisfies these requirements, then $i$ needs only knowledge of the states of agents within $M_{i}$ at each time instant to fulfil the collision avoidance objective. The fourth requirement also guarantees that $\sum_{j \in M_{i}} \frac{\partial V_{i j}}{\partial q_{i}}=\sum_{j \neq i} \frac{\partial V_{i j}}{\partial q_{i}}$. The gradient with respect to $q$ and the partial derivative of $V_{i j}$ with respect to $q_{i}$ are computed by $\nabla V_{i j}=2 \rho_{i j} D_{i j} q$ and $\frac{\partial V_{i j}}{\partial q_{i}}=2 \rho_{i j}\left(D_{i j}\right)_{i} q$ where the matrices $D_{i j},\left(D_{i j}\right)_{i}$, for $i<j$ are given by $D_{i j}=\tilde{D}_{i j} \otimes I_{2}$, where $\left(\tilde{D}_{i j}\right)_{i i}=\left(\tilde{D}_{i j}\right)_{j j}=1,\left(\tilde{D}_{i j}\right)_{i j}=\left(\tilde{D}_{i j}\right)_{j i}=$ -1 and $\left(\tilde{D}_{i j}\right)_{k l}=0$ for $k, l \neq i, j$, and $\left(D_{i j}\right)_{i}=$ $\left[\begin{array}{lllll}O_{1 \times(i-1)} & 1 & O_{1 \times(j-i-1)} & -1 & O_{1 \times(N-j)}\end{array}\right] \otimes I_{2}$. The definition of $D_{i j},\left(D_{i j}\right)_{i}$ for $i>j$ is straightforward.This definition of $V_{i j}$ guarantees that the potential field has the following symmetry property: $\rho_{i j}=\rho_{j i}, \forall i, j \in \mathcal{N}, i \neq j$.

For the purpose of aggregation, we define an attractive potential $W_{i j}: \mathbb{R}^{2} \rightarrow \mathbb{R}_{+}$between agents $i$ and $j \in N_{i}$, which is required to have the following properties: (1) $W_{i j}$ is a function of the square norm of the distance between $i, j$, i.e. $W_{i j}=W_{i j}\left(\left\|q_{i}-q_{j}\right\|^{2}\right)=W_{i j}\left(\beta_{i j}\right)$, (2) $W_{i j}$ is defined on $\beta_{i j} \in\left[0, d^{2}\right)$, (3) $W_{i j} \rightarrow \infty$ whenever $\beta_{i j} \rightarrow d^{2}$, (4) it is $C^{1}$ for $\beta_{i j} \in\left[0, d^{2}\right)$ and (5) the term $p_{i j} \triangleq \frac{\partial W_{i j}}{\partial \beta_{i j}}$ satisfies $p_{i j}>0$ for $0 \leq \beta_{i j}<d^{2}$. Function $W_{i j}$ is hence defined to ensure that the agents that are located at a distance no larger than $d$ from agent $i$ at time $t=0$, remain within agent $i$ 's sensing zone for all $t>0$. We also have $\nabla W_{i j}=2 p_{i j} D_{i j} q$ and $\frac{\partial W_{i j}}{\partial q_{i}}=2 p_{i j}\left(D_{i j}\right)_{i} q$ where $p_{i j} \triangleq \frac{\partial W_{i j}}{\partial \beta_{i j}}$ and the matrices $D_{i j},\left(D_{i j}\right)_{i}$ were defined previously. The following symmetry property holds in this case as well: $p_{i j}=p_{j i}, \forall j \in N_{i}$.

The proposed controller for agent $i$ is given as the sum of the negative gradients of the two potentials in the $q_{i}$ direction:

$$
u_{i}=-\sum_{j \in N_{i}} \frac{\partial W_{i j}}{\partial q_{i}}-\sum_{j \in M_{i}} \frac{\partial V_{i j}}{\partial q_{i}}
$$

The control law can also be written as $u_{i}=$ $-2 \sum_{j \in N_{i}} p_{i j}\left(q_{i}-q_{j}\right)-2 \sum_{j \in M_{i}} \rho_{i j}\left(q_{i}-q_{j}\right)$. Since (3) requires knowledge only of the states of agents belonging to $N_{i} \cup M_{i}$, it respects the agents' limited sensing specifications. It is hence clearly a distributed control law. This control also guarantees the following for the closed loop system: (i) since the repulsive potential tends to infinity whenever two agents collide, its negative gradient, imposed on the motion of each agent, guarantees collision avoidance and (ii) for the same reasons, each agent $j$ initially located at a distance less than $d$ from $i$, will never leave the sensing zone of $i$. These are proven explicitly in the next section.

\section{Stability Analysis}

The function $V=\sum_{i}\left(\sum_{j \in N_{i}} W_{i j}+\sum_{j \neq i} V_{i j}\right)$ is used as a candidate Lyapunov function for the multi-agent system. Differentiating $V$ we get $\dot{V}=(\nabla V)^{T} \cdot \dot{q}$. We also have $\sum_{i} \sum_{j \in N_{i}} \nabla W_{i j}=2 \sum_{i} \sum_{j \in N_{i}} p_{i j} D_{i j} q=4\left(P \otimes I_{2}\right) q$ where the $N \times N$ matrix $P$ can shown to be given by $P_{i i}=\sum_{j \in N_{i}} p_{i j}$, $P_{i j}=-p_{i j}$ for $j \in N_{i}, i \neq j$, and $P_{i j}=0$ for $j \notin N_{i}$. We can also compute $\sum_{i} \sum_{j \neq i} \nabla V_{i j}=2 \sum_{i} \sum_{j \neq i} \rho_{i j} D_{i j} q=$ $2\left(R_{1} \otimes I_{2}\right) q$ where matrix $R_{1}$ can be computed by $\left(R_{1}\right)_{i i}=$ $\sum_{j \neq i} \rho_{i j}+\sum_{j \neq i} \rho_{j i}$ and $\left(R_{1}\right)_{i j}-\rho_{i j}-\rho_{j i}$, for $i \neq j$. We hence get $\nabla V=4\left(P \otimes I_{2}\right) q+2\left(R_{1} \otimes I_{2}\right) q$. The time derivative of the stack vector of the agents'positions can be shown to be given by $\dot{q}=-2\left(P \otimes I_{2}\right) q-2\left(R \otimes I_{2}\right) q$, where the matrix $R$ is given by $R_{i i}=\sum_{j \neq i} \rho_{i j}$ and $R_{i j}=-\rho_{i j}$, for $i \neq j$. Hence $\dot{q}=-2\left(P \otimes I_{2}\right) q-$ $2\left(R \otimes I_{2}\right) q$. Using now $\rho_{i j}=\rho_{j i}$ we get $R_{1}=2 R$ so that $\dot{V}=(\nabla V)^{T} \cdot \dot{q}=-\left(4\left(P \otimes I_{2}\right) q+2\left(R_{1} \otimes I_{2}\right) q\right)^{T}$.

$$
\begin{aligned}
\cdot\left(2\left(P \otimes I_{2}\right) q+2\left(R \otimes I_{2}\right) q\right) & \stackrel{R_{1}=2 R}{\Rightarrow} \\
& \Rightarrow \dot{V}=-8\left\|\left(\left(P \otimes I_{2}\right) q+\left(R \otimes I_{2}\right) q\right)\right\|^{2} \leq 0
\end{aligned}
$$

We now state the first result of this paper:

Theorem 1: Assume that the swarm (1) evolves under the control law (3). Then the system reaches a configuration in which $u=0$, i.e. $u_{i}=0$ for all $i \in \mathcal{N}$. 
Proof: The level sets of $V$ are compact and invariant wrt the relative positions of adjacent agents. Specifically, the set $\Omega_{c}=\{q: V(q) \leq c\}$ for $c>0$ is closed by continuity of $V$. We have $V \leq c \Rightarrow W_{i j} \leq c \Rightarrow\left\|q_{i}-q_{j}\right\| \leq \sqrt{W_{i j}^{-1}(c)}$, $\forall(i, j) \in E$. LaSalle's principle and (4) guarantee that the system converges to the largest invariant subset of the set $S=\left\{q:\left((P+R) \otimes I_{2}\right) q=0\right\}$. Since $u=\dot{q}=$ $-2\left(P \otimes I_{2}\right) q-2\left(R \otimes I_{2}\right) q$, we have $u=0 . \diamond$

The next Lemma guarantees collision avoidance:

Lemma 2: Consider the system (1) driven by the control (3) and starting from a feasible set of initial conditions $\mathcal{I}(q)=\left\{q \mid\left\|q_{i}-q_{j}\right\|>0, \forall i, j \in \mathcal{N}, i \neq j\right\}$. Then $\mathcal{I}(q)$ is invariant for the trajectories of the closed loop system.

Proof: For every initial condition $q(0) \in \mathcal{I}(q)$, the time derivative of $V$ remains non-positive for all $t \geq 0$, by virtue of (4). Hence $V(q(t)) \leq V(q(0))<\infty$ for all $t \geq 0$. Since $V \rightarrow \infty$ when $\left\|q_{i}-q_{j}\right\| \rightarrow 0$ for at least one pair $i, j \in \mathcal{N}$, we conclude that $q(t) \in \mathcal{I}(q)$, for all $t \geq 0$. $\diamond$

The next result states that the proposed control forces agents that are initially located within distance $d$ from each other to remain within this distance for all time. Hence the definition of $N_{i}$ is rendered meaningful since each agent $i$ does not have to violate its sensing constraints in order to sense agents within $N_{i}$ as the closed loop system evolves. Thus, the control law guarantees that an agent $j$ initially located at a distance less than $d$ from $i$, will never leave the sensing zone of $i$. This is proved in the following:

Lemma 3: Consider the system (1) driven by the control (3). The set $\mathcal{J}(q)=\left\{q \mid\left\|q_{i}-q_{j}\right\|<d, \forall(i, j) \in E\right\}$ is invariant for the trajectories of the closed loop system.

Proof: Since $V(q(t)) \leq V(q(0))<\infty$ for all $t \geq 0$ and $V \rightarrow \infty$ when $\left\|q_{i}-q_{j}\right\| \rightarrow d$ for at least one pair $(i, j) \in E$, we conclude that $q(t) \in \mathcal{J}(q)$, for all $t \geq 0$. $\diamond$

Based on the fact that all agents initially located within distance $d$ from each other remain within this distance for all time, the set $N_{i}$ is static. Hence no new edges are created even when an agent not initially located within the sensing zone of another, enters inside it at some time instant $t>0$. The case of dynamic edge addition, i.e. adding new edges to the communication graph each time a new agent enters the sensing zone of another, is treated in Section VI. In essence, starting from $\mathcal{J}(q) \cup \mathcal{I}(q)$, the communication graph remains invariant (no edges are lost) and collisions are avoided.

In the sequel, we derive bounds on the swarm dispersion. We first show that the "swarm center" $\bar{q} \triangleq$ $\frac{1}{N} \sum_{i=1}^{N} q_{i}$ remains constant, i.e. $\bar{q}(t)=\bar{q}(0)$ for all $t \geq$ 0 . This is proven by the fact that $\dot{\bar{q}}=\frac{1}{N} \sum_{i=1}^{N} \dot{q}_{i}=$ $-\frac{2}{N} \sum_{i=1}^{N}\left(\sum_{j \in M_{i}} \rho_{i j}\left(q_{i}-q_{j}\right)+\sum_{j \in N_{i}} p_{i j}\left(q_{i}-q_{j}\right)\right)=0$. Since $\bar{q}$ is constant, we assume without loss of generality that it is the origin of the coordinate system, i.e. $\bar{q}=0$.

Moreover, at an equilibrium point we have $u=$ 0 , by virtue of Theorem 1. Considering the function $\Phi=\frac{1}{2} \sum_{i} q_{i}^{T} q_{i}$ and taking its time derivative we have
$\Phi=\frac{1}{2} \sum_{i} q_{i}^{T} q_{i} \Rightarrow \dot{\Phi}=\sum_{i} q_{i}^{T} \dot{q}_{i}=0$. Thus, $\dot{\Phi}=$ $-2 \sum_{i}\left(q_{i}^{T}\left(\sum_{j \in M_{i}} \rho_{i j}\left(q_{i}-q_{j}\right)+\sum_{j \in N_{i}} p_{i j}\left(q_{i}-q_{j}\right)\right)\right)=$ $-\sum_{i}\left(\sum_{j \in M_{i}} \rho_{i j}\left\|q_{i}-q_{j}\right\|^{2}+\sum_{j \in N_{i}} p_{i j}\left\|q_{i}-q_{j}\right\|^{2}\right)=0$ and hence at an equilibrium position:

$$
\sum_{i} \sum_{j \in N_{i}} p_{i j}\left\|q_{i}-q_{j}\right\|^{2}=\sum_{i} \sum_{j \in M_{i}}\left|\rho_{i j}\right|\left\|q_{i}-q_{j}\right\|^{2}
$$

since $\rho_{i j} \leq 0, \forall j \in M_{i}$. The last equation allows us to derive bounds on the swarm size. These are based on the bounds on the designed potentials. The attractive potential is chosen so that $p_{i j} \geq a$ where $a>0$. We then have $\sum_{i} \sum_{j \in N_{i}} p_{i j}\left\|q_{i}-q_{j}\right\|^{2} \geq a \sum_{i} \sum_{j \in N_{i}}\left\|q_{i}-q_{j}\right\|^{2}$. The repulsive potential is chosen so that $\left|\rho_{i j}\right| \leq \frac{\rho}{\beta_{i j}}$ where $\rho>0$. Then, $\sum_{i} \sum_{j \in M_{i}}\left|\rho_{i j}\right|\left\|q_{i}-q_{j}\right\|^{2} \leq \rho \sum_{i}\left|M_{i}\right|$ where $\left|M_{i}\right|$ is the cardinality of $M_{i}$. Eq. (5) yields $\sum_{i} \sum_{j \in N_{i}}\left\|q_{i}-q_{j}\right\|^{2}=$ $\sum_{i} \sum_{j \in N_{i}} \beta_{i j} \leq \frac{\rho}{a} \sum_{i}\left|M_{i}\right|$. The right hand side is maximized whenever each agent is found at a distance less than $d_{1}$ from all others, i.e. the repulsive potential is active for all pairs $i, j \in \mathcal{N}$. We then have $\sum_{i}\left|M_{i}\right| \leq N(N-1)$. For each pair of agents that form an edge, we then have: $\beta_{i j} \leq \frac{\rho}{a} N(N-1), \forall(i, j) \in E$. We then have:

Theorem 4: Assume that the swarm (1) evolves under the control law (3) and the initially formed communication graph is connected. Denote by $\beta_{\max }$ the maximum distance between two members of the group, i.e. $\beta_{\max }=$ $\max _{i, j \in \mathcal{N}}\left\|q_{i}-q_{j}\right\|^{2}$. Under the preceding assumptions, the following bound holds at steady state: $\beta_{\max } \leq \frac{\rho}{a} N(N-1)^{2}$. Proof: Since the graph is connected, the maximum length of a path connecting any two vertices is $N-1$. The result now follows from the fact that $\beta_{i j} \leq \frac{\rho}{a} N(N-1), \forall(i, j) \in E . \diamond$

\section{THE CASE OF NONHOLONOMIC KINEMATIC UNICYCLE-TYPE AGENTS}

In this section, the extension of the proposed framework to the nonholonomic unicycle case is presented. We consider $N$ nonholonomic agents operating in $W \subset \mathbb{R}^{2}$. Let $q_{i}=\left[x_{i}, y_{i}\right]^{T} \in \mathbb{R}^{2}$ denote the position of agent $i$. The configuration space is spanned by $q=\left[q_{1}^{T}, \ldots, q_{N}^{T}\right]^{T}$. Each agent has a specific orientation $\theta_{i}$ wrt the global coordinate frame. The orientation vector of the agents is given by $\theta=\left[\theta_{1} \ldots \theta_{N}\right]^{T}$. The configuration of each agent is given by $p_{i}=\left[\begin{array}{ll}q_{i} & \theta_{i}\end{array}\right] \in \mathbb{R}^{2} \times(-\pi, \pi]$. The motion of the agents is described by the following nonholonomic kinematics:

$$
\dot{x}_{i}=u_{i} \cos \theta_{i}, \dot{y}_{i}=u_{i} \sin \theta_{i}, \dot{\theta}_{i}=\omega_{i}, i \in \mathcal{N}=[1, \ldots, N]
$$

where $u_{i}, \omega_{i}$ denote the translational and rotational velocity of agent $i$, respectively. Similarly to the previous case, the control law is of the form $u_{i}=u_{i}\left(p_{i}, p_{j}\right), \omega_{i}=$ $\omega_{i}\left(p_{i}, p_{j}\right), j \in N_{i} \cup M_{i}, i \in \mathcal{N}$. We consider again the 
function $V=\sum_{i}\left(\sum_{j \in N_{i}} W_{i j}+\sum_{j \neq i} V_{i j}\right)$ as a candidate Lyapunov function. Since the proposed control law will be discontinuous we use the extension of LaSalle's Principle for nonsmooth systems of [10] for the time derivative of the candidate Lyapunov function. Since $V$ is smooth we have $\partial V=\{\nabla V\}$, where $\partial V$ is the generalized gradient of $V$ [10], and is given by $\nabla V=4\left(P \otimes I_{2}\right) q+2\left(R_{1} \otimes I_{2}\right) q=$ $4\left(\left(P \otimes I_{2}\right) q+\left(R \otimes I_{2}\right) q\right)$. We define $P+R \triangleq F$. We denote the stack vector $q=[x, y]^{T}$ into the coefficients that correspond to the $x, y$ directions of the agents, and $(a)_{i}$ denotes the $i$-th element of a vector $a$. The following holds:

Theorem 5: Assume that (6) evolves under

$$
\begin{gathered}
u_{i}=-\operatorname{sgn}\left\{f_{x i} \cos \theta_{i}+f_{y i} \sin \theta_{i}\right\} \cdot\left(f_{x i}^{2}+f_{y i}^{2}\right) \\
\omega_{i}=-\left(\theta_{i}-\arctan 2\left(f_{y i}, f_{x i}\right)\right)
\end{gathered}
$$

where $(F x)_{i}=f_{x i},(F y)_{i}=f_{y i}$. Then the system reaches the equilibrium points of the single integrator case, i.e. a configuration in which $\left(\left(P \otimes I_{2}\right)+\left(R \otimes I_{2}\right)\right) q=0$.

Proof: The generalized time derivative of $V$ is calculated by $\dot{\tilde{V}} \subset \sum_{i \in N}\left\{4 K\left[u_{i}\right]\left((F x)_{i} \cos \theta_{i}+(F y)_{i} \sin \theta_{i}\right)\right\}$, where we used Theorem 1.3 in [9] to calculate the inclusions of the Filippov set $K[$.$] in the previous analysis. Since K[\operatorname{sgn}(x)] x=$ $\{|x|\}([9]$, Theorem 1.7), the choice (7),(8) results in $\dot{\widetilde{V}}=$ $-\sum_{i}\left\{4\left|f_{x i} \cos \theta_{i}+f_{y i} \sin \theta_{i}\right|\left(f_{x i}^{2}+f_{y i}^{2}\right)^{1 / 2}\right\} \leq 0$, so that the generalized derivative of $V$ reduces to a singleton. By the nonsmooth version of LaSalle's Invariance principle [10], the agents converge to the largest invariant subset of the set $S=$ $\left\{\left(f_{x i}=f_{y i}=0\right) \vee\left(f_{x i} \cos \theta_{i}+f_{y i} \sin \theta_{i}=0\right), \forall i \in N\right\}$.

However, for each $i \in N$, we have $\left|\omega_{i}\right|=\frac{\pi}{2}$ whenever $f_{x i} \cos \theta_{i}+f_{y i} \sin \theta_{i}=0$, due to (8). In particular [11], this choice of angular velocity renders the surface $f_{x i} \cos \theta_{i}+f_{y i} \sin \theta_{i}=0$ repulsive for agent $i$, whenever $i$ is not located at the desired equilibrium, namely when $f_{x i}=f_{y i}=0$. Hence the largest invariant set $E$ contained in $S$ is $S \supset E=\left\{f_{x i}=f_{y i}=0, \forall i \in N\right\}$ which is equivalent to the equilibria of the single integrator case: $\left(\left(P \otimes I_{2}\right)+\left(R \otimes I_{2}\right)\right) q=0 . \diamond$

Hence the control (7),(8) forces the nonholonomic team to behave exactly the same as in the single integrator case.

\section{The CASE of Dynamic GRaphS}

The previous sections involved the case where the communication graph considered was static, i.e. no new edges were added whenever an agent, not initially located within the sensing zone of another, entered its sensing zone. We now consider creation of new edges whenever an agent enters the sensing zone of another. This naturally leads to a smaller swarm size and corresponds to a more realistic formulation of the problem in hand. The results of this section can also be applied to the nonholonomic case.

We consider two types of communication sets for each agent $i$ at each time instant. The first corresponds to the sensing zone of $i$, i.e. to the agents that $i$ senses at each time instant: $N_{i}(t)=\left\{j \in N, j \neq i:\left\|q_{i}(t)-q_{j}(t)\right\|<d\right\}$. In order to add new communication links, we assume that a new edge is created whenever a new agent enters a subset of the sensing zone of $i$ at some time instant. Specifically, we define the set: $N_{i}^{*}(t)=\left\{j \in N, j \neq i:\left\|q_{i}(t)-q_{j}(t)\right\| \leq d-\varepsilon\right\}$, where $0<\varepsilon<<d$. Thus, $N_{i}^{*}(t) \subseteq N_{i}(t)$. We assume that the communication graph is initially formed based on $N_{i}(0)$,i.e. $E(0)=\left\{(i, j): j \in N_{i}(0)\right\}$. The set of edges is updated as: $E(t)=E\left(t^{-}\right) \cup E^{*}(t)$, where $E^{*}(t)=$ $\left\{(i, j):\left((i, j) \notin E\left(t^{-}\right)\right) \wedge\left(j \in N_{i}^{*}(t)\right)\right\}$. Thus, a new edge is added when an agent $j$, that didn't form an edge with $i$, enters at some time $t$ the set $N_{i}^{*}(t)$ which is a subset of $i$ 's sensing zone. By designing the control in such a way to force agents that come to a distance $d-\varepsilon$ between them, to remain within distance $d$ for all time afterwards, this definition of edge addition becomes meaningful since each agent has to sense only agents within its sensing zone at each time instant.

The main difference with the the static graph case is the definition of the aggregation potential between $i$ and $j$. We denote this potential in the dynamic graph case by $W_{i j}^{d}$ and recalling the definition of $W_{i j}$ in the static case, $W_{i j}^{d}$ is defined as: $W_{i j}^{d}\left(\beta_{i j}\right)=W_{i j}\left(\beta_{i j}\right)$, for $(i, j) \in E(t)$ and $W_{i j}^{d}\left(\beta_{i j}\right)=\tilde{W}_{i j}\left(\beta_{i j}\right)$, for $(i, j) \notin E(t)$. Hence when two agents form an edge, their aggregation potential is identical to the the static case. Whenever an agent $j$ forms a new edge with $i$, the function $W_{i j}^{d}$ switches from $\tilde{W}_{i j}$ to $W_{i j}$. The function $\tilde{W}_{i j}$ is defined so that the switch to $W_{i j}$ is held in a sufficiently smooth manner. This is encoded in the following properties: (1) $\tilde{W}_{i j}\left(\beta_{i j}\right)=W_{i j}\left(\beta_{i j}\right)$, for $\left\|q_{i}(t)-q_{j}(t)\right\| \leq d-\varepsilon$, (2) $\tilde{W}_{i j}\left(\beta_{i j}\right)=\tilde{W}_{i j}\left(d^{2}\right)=$ cnst, for $\left\|q_{i}(t)-q_{j}(t)\right\|>d$, (3) $W_{i j}\left((d-\varepsilon)^{2}\right)=$ $\tilde{W}_{i j}\left((d-\varepsilon)^{2}\right)$ and $\frac{\partial W_{i j}}{\partial \beta_{i j}}\left((d-\varepsilon)^{2}\right)=\frac{\partial \tilde{W}_{i j}}{\partial \beta_{i j}}\left((d-\varepsilon)^{2}\right)$, (4) $\tilde{W}_{i j}$ is everywhere $C^{1}$, and (5) $\frac{\partial \tilde{W}_{i j}}{\partial \beta_{i j}}>0$ for $d-\varepsilon<$ $\left\|q_{i}(t)-q_{j}(t)\right\|<d$. The control law is now:

$$
u_{i}=-\sum_{(i, j) \in E} \frac{\partial W_{i j}}{\partial q_{i}}-\sum_{(i, j) \notin E} \frac{\partial \tilde{W}_{i j}}{\partial q_{i}}-\sum_{j \in M_{i}} \frac{\partial V_{i j}}{\partial q_{i}}
$$

This definition of $W_{i j}^{d}$ allows $i$ to neglect agents outside its sensing zone. Moreover, $V_{i j}$ is the same as in the static graph case. The overall system is a hybrid system in which discrete transitions occur each time a new edge is added, i.e. each time two agents not forming an edge before come to a distance closer than $d-\varepsilon$ from one another. Convergence is checked using the common Lyapunov function tool from hybrid stability theory [6]. In particular, the function $V=\sum_{i} \sum_{j \neq i}\left(W_{i j}^{d}+V_{i j}\right)$ serves as a valid common Lyapunov function for the underlying hybrid system. Using the analysis of the single integrator case, it is easy to show that at time spaces where no new edges are added, the time derivative of $V$ is given by $\dot{V}=-8\left\|\left(\left(P^{d} \otimes I_{2}\right) q+\left(R \otimes I_{2}\right) q\right)\right\|^{2} \leq$ 0 , where the $P^{d}$ matrix is defined as $P_{i i}^{d}=\sum_{j \neq i} p_{i j}^{d}$ and $P_{i j}^{d}=-p_{i j}^{d}$, for $i \neq j$, with $p_{i j}^{d}=\frac{\partial W_{i j}}{\partial \beta_{i j}}$,for $(i, j) \in E$ and $p_{i j}^{d}=\frac{\partial \tilde{W}_{i j}}{\partial \beta_{i j}}$, for $(i, j) \notin E$. At times when new edge are added, the common Lyapunov function and the 
control laws of all agents are continuously differentiable while the values of $V$, its time derivative, and the values of the control laws remain constant. Hence $V$ serves as a common Lyapunov function and since no Zeno behavior occurs whenever the system enters a new discrete state, i.e. once an edge is added it is never deleted, we quote the LaSalle's Principle for Hybrid Systems [6] to show that the system converges to the largest invariant subset of the set $S=\left\{q:\left(\left(P^{d}+R\right) \otimes I_{2}\right) q=0\right\}$. Thus, the results of Theorem 1 and Lemma 2 hold in this case as well. The counterpart of Lemma 3 in the dynamic graph case involves the fact that once an agent $j$ enters the set $N_{i}^{*}(t)$ for the first time, it is forced to remain within the sensing zone of $i$, encoded by the set $N_{i}(t)$, for all future times. Thus, the definition of edges in the dynamic graph case is meaningful since it respects the sensing capabilities of all agents. The following counterpart of Lemma 3 holds:

Lemma 6: Consider the system (1) driven by the control law (9). Then, all agent pairs that come into distance less or equal to $d-\varepsilon$ for the first time, remain within distance strictly less than $d$ for all future times.

Proof: Since $V(q(t)) \leq V(q(0))<\infty$ for all $t \geq 0$ and $V \rightarrow \infty$ when $\left\|q_{i}-q_{j}\right\| \rightarrow d$ for at least one pair of agents $(i, j)$ that either (i) have formed an edge at $t=0$, or (ii) have formed an edge at some time $\tau, 0 \leq \tau \leq t$ we conclude that all pairs of agents that did not initially form an edge and come to a distance less than $d-\varepsilon$ for the first time, remain within distance strictly less than $d$ for all future times. $\diamond$

The fact that agents that initially formed edges remain within distance strictly less than $d$ from each other is proven in Lemma 3. These two Lemmas guarantee that the definition of edges in the dynamic graph case respects the limited sensing capabilities of all agents, since each agent has to sense only agents within its sensing zone in order to fulfill the communication link imposed by the existence of edges.

Having now established a framework that allows for addition of edges in communication graph while maintaining connectivity, we can follow the analysis of the static case to show that similar bounds for the swarm size can be derived in this case as well. In particular, the system now reaches a configuration where equation $\left(\left(P^{d}+R\right) \otimes I_{2}\right) q=0$ holds. Following the analysis of the static graph case, an equation similar to (5) is derived in the dynamic graph case as well:

$$
\sum_{i} \sum_{j \neq i} p_{i j}^{d}\left\|q_{i}-q_{j}\right\|^{2}=\sum_{i} \sum_{j \in M_{i}}\left|\rho_{i j}\right|\left\|q_{i}-q_{j}\right\|^{2}
$$

An improved result on the bound of the swarm size with respect to the static graph case can be obtained in the dynamic graph case. In particular, using the notation $\left\|q_{i}-q_{j}\right\|^{2}=\beta_{i j}$, the last equation can be rewritten as $\sum_{i}\left(\sum_{j \in M_{i}} p_{i j}^{d} \beta_{i j}+\sum_{j \notin M_{i}} p_{i j}^{d} \beta_{i j}\right)=\sum_{i} \sum_{j \in M_{i}}\left|\rho_{i j}\right| \beta_{i j} \Rightarrow$ $\sum_{i} \sum_{j \notin M_{i}} p_{i j}^{d} \beta_{i j}=\sum_{i} \sum_{j \in M_{i}}\left(\left|\rho_{i j}\right|-p_{i j}^{d}\right) \beta_{i j}$. Assuming that the repulsion term satisfies the bound $\left|\rho_{i j}\right| \leq \frac{\rho}{\beta_{i j}}$ and noting that $p_{i j}^{d} \geq 0$ for all $i, j \in \mathcal{N}$, we get $\sum_{i} \sum_{j \in M_{i}}\left(\left|\rho_{i j}\right|-p_{i j}^{d}\right) \beta_{i j} \leq \sum_{i} \sum_{j \in M_{i}}\left|\rho_{i j}\right| \beta_{i j} \leq \rho \sum_{i}\left|M_{i}\right|$, so that $\sum_{i} \sum_{j \notin M_{i}} p_{i j}^{d} \beta_{i j} \leq \rho \sum_{i}\left|M_{i}\right|$. We now denote by $E(\infty)$ the set of edges that have been formed at steady state. Using the bound $p_{i j}>a$ on the attractive term for the agents that have formed an edge the left hand side of the previous inequality can be shown to by bounded as follows: $\sum_{i} \sum_{j \notin M_{i}} p_{i j}^{d} \beta_{i j} \geq \sum_{i} \sum_{j:(i, j) \in E(\infty)} a d_{1}^{2}$. The last two bounds suggest that $\sum_{i} \sum_{\substack{j:(i, j) \in E(\infty) \\ j \notin M_{i}}}{ }^{j \notin M_{i}} d d_{1}^{2} \leq \rho \sum_{i}\left|M_{i}\right|$ at steady state. We will now show that an appropriate choice of $d_{1}$ forces all agents that have formed an edge to be at a distance not larger than $d_{1}$ at steady state, i.e. $\left\|q_{i}-q_{j}\right\| \leq d_{1}$, for all $(i, j) \in E(\infty)$. This is proved by showing that the inequality $\sum_{i} \sum_{\substack{j:(i, j) \in E(\infty) \\ j \notin M_{i}}} a d_{1}^{2} \leq \rho \sum_{i}\left|M_{i}\right|$ is not viable even in the worst case scenario. Thus, let us assume that at least one pair that has formed an edge at steady state is at a distance larger than $d_{1}$ from one another, i.e. $\left\|q_{k}-q_{l}\right\|>d_{1}$ for some $(k, l) \in E(\infty)$. This implies that $k \notin M_{l}$ and vice verca. In that case $\sum_{i} \sum_{\substack{j:(i, j) \in E(\infty) \\ j \notin M_{i}}} a d_{1}^{2} \leq \rho \sum_{i}\left|M_{i}\right|$ yields $\sum_{i} \sum_{j:(i, j) \in E(\infty)} a d_{1}^{2}=2 a d_{1}^{2 \notin} \leq \sum_{i} \sum_{j \notin M_{i}} p_{i j}^{d} \beta_{i j} \leq \rho \sum_{i}\left|M_{i}\right|=$ $j \notin M_{i}$

$\rho\{(N-1)(N-2)+2(N-2)\}=\rho\left(N^{2}-N-2\right) \Rightarrow$ $d_{1}^{2} \leq \frac{\rho\left(N^{2}-N-2\right)}{2 a d_{1}^{2}}$. The last inequality is rendered impossible by choosing $d_{1}^{2}>\frac{\rho\left(N^{2}-N-2\right)}{2 a}$. In this case, we have $j \in M_{i}$ for all pairs of agents that form an edge at steady state, and hence an ultimate bound is: $\beta_{i j} \leq d_{1}^{2}, \forall(i, j) \in E(\infty)$. This equation provides the means to provide a better bound of the swarm size, as will be shown in the sequel. We first note that $d_{1}$ can be chosen by the following inequality: $\frac{\rho\left(N^{2}-N-2\right)}{2 a}<d_{1}^{2}<\frac{\rho N(N-1)}{a}$. The last inequality is viable since the inequality $\frac{\rho\left(N^{2}-N-2\right)}{2 a}<\frac{\rho N(N-1)}{a}$ is equivalent to $N^{2}-N+2>0$ which holds for all $N>0$. The following Theorem, which is the counterpart of Theorem 4 in the dynamic graph case, shows that a better bound is derived in the dynamic graph case:

Theorem 7: Assume that the swarm (1) evolves under the control law (9) and the initially formed communication graph is connected. Denote by $\beta_{\max }^{d}$ the maximum distance between two members of the group, i.e. $\beta_{\max }^{d}=$ $\max _{i, j \in \mathcal{N}}\left\|q_{i}-q_{j}\right\|^{2}$. Assume that the parameter $d_{1}$ satisfies $\frac{\rho\left(N^{2}-N-2\right)}{2 a}<d_{1}^{2}<\frac{\rho N(N-1)}{a}$. Under the preceding assumptions, the following bound holds at steady state: $\beta_{\max }^{d} \leq$ $d_{1}^{2}(N-1)$. We moreover have $\beta_{\max }^{d}<\beta_{\max }$ where $\beta_{\max }=$ $\frac{\rho}{a} N(N-1)^{2}$ is the swarm size corresponding to the static graph case of Theorem 4.

Proof: Since the graph is connected, the maximum length of a path connecting two arbitrary vertices is $N-1$. Equation $\beta_{i j} \leq d_{1}^{2}, \forall(i, j) \in E(\infty)$ now yields $\beta_{\max }^{d} \leq d_{1}^{2}(N-1)$. 
Now since $d_{1}$ satisfies the bounds assumed, we have $\beta_{\text {max }}^{d} \leq$ $d_{1}^{2}(N-1)<\frac{\rho N(N-1)^{2}}{a}=\beta_{\max } \Rightarrow \beta_{\max }^{d}<\beta_{\max } \cdot \diamond$

This result shows that allowing edges to be added in a dynamic fashion, leads to an improved (i.e. smaller) swarm size. This derivation is not surprising, since the addition of new communication links increases the attractive potential and hence leads to a tighter swarm size.

\section{Simulations}

The derived results are now supported through a series of computer simulations.

The first simulation of Figure 1 involves a swarm of nine single integrator agents navigating under the proposed control in both the static and dynamic edge addition cases. In both cases the agents have the same initial conditions and controller parameters. The first graph shows the agents' initial positions. In the first case on the left they navigate under (3) while in the second case on the right under (9). As seen in Figure 1 the latter control law leads to a tighter final swarm size, something also shown in the comparison of the final swarm sizes of the two case in Figure 2. This Figure shows the evolution of the swarm size in both cases from time 1000 and onwards. It can be shown that apart from the reduce of the swarm size, the convergence rate is also increased in the case of the dynamic edge addition. This is witnessed by the significantly smaller swarm size the team has attained at time 1000 in the second case.
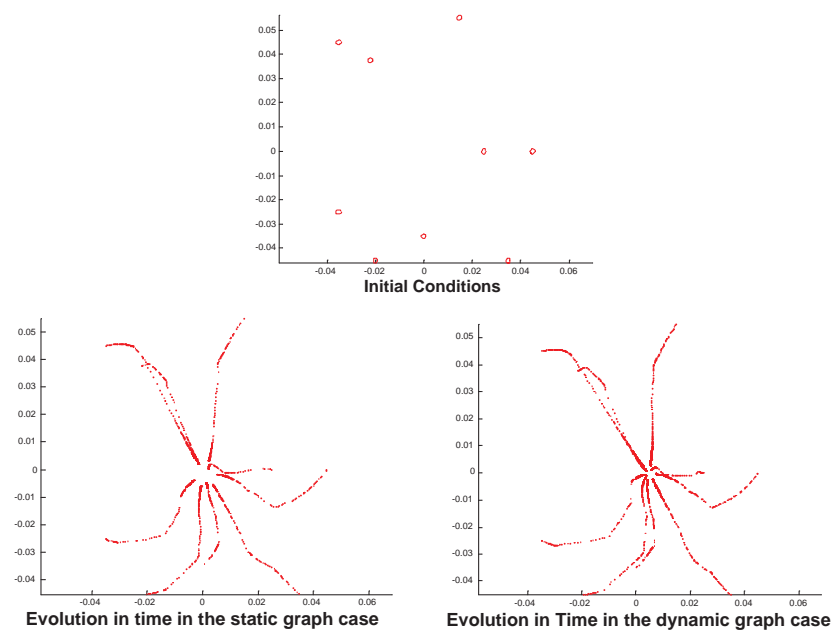

Fig. 1. Evolution in time of the swarm under the control law (3), for the static communication graph case, on the left, and the control law (9), for the dynamic graph case, on the right. The communication graph is connected in both cases. The second control law leads to a tighter swarm size.

The second simulation in Figure 3 involves evolution of a swarm of six unicycles navigating under $(7,8)$. The first graph shows the initial positions of the six agents while the second one the evolution of their trajectories. Swarm aggregation is eventually achieved, since the communication graph that is formed based on the initial relative positions of the agents, is connected. The same values of controller parameters as in the first simulation have been retained in the simulation of Figure 3 as well.
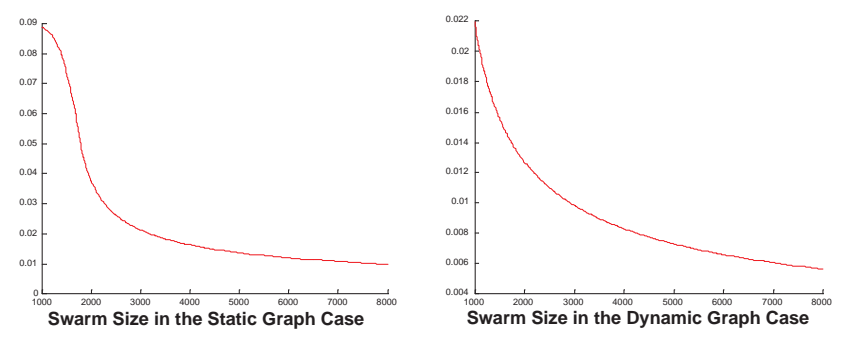

Fig. 2. Evolution of the swarm size for the two simulations of Figure 1. The dynamic graph formulation leads to a smaller swarm size.
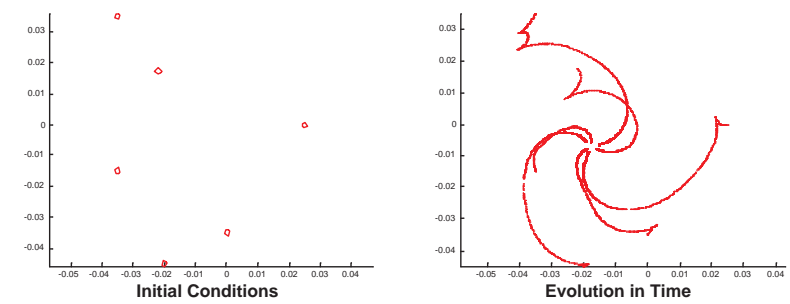

Fig. 3. Evolution in time of the nonholonomic swarm under the control laws $(7,8)$. The communication graph is connected.

\section{CONCLUSIONS}

A distributed control strategy for connectivity preserving swarm aggregation with collision avoidance was presented. In the case of dynamic edge addition, an improved bound on the swarm size was derived. The results were extended to deal with the case of nonholonomic agents as well.

\section{REFERENCES}

[1] M. Arcak. Passivity as a design tool for group coordination. IEEE Transactions on Automatic Control, 52(2):1380-1390, 2007.

[2] D. V. Dimarogonas, S. G. Loizou, K.J. Kyriakopoulos, and M. M. Zavlanos. A feedback stabilization and collision avoidance scheme for multiple independent non-point agents. Automatica, 42(2):229243, 2006.

[3] V. Gazi and K.M. Passino. Stability analysis of swarms. IEEE Transactions on Automatic Control, 48(4):692-696, 2003.

[4] A. Jadbabaie, J. Lin, and A.S. Morse. Coordination of groups of mobile autonomous agents using nearest neighbor rules. IEEE Transactions on Automatic Control, 48(6):988-1001, 2003.

[5] M. Ji and M. Egerstedt. Connectedness preserving distibuted coordination control over dynamic graphs. 2005 American Control Conference, pages $93-98$.

[6] J. Lygeros, K.H. Johansson, S. Simic, J. Zhang, and S. Sastry. Dynamical properties of hybrid automata. IEEE Transactions on Automatic Control, 48(1):2-17, 2003.

[7] G. Notarstefano, K. Savla, F. Bullo, and A. Jadbabaie. Maintaining limited-range connectivity among second-order agents. 2006 American Control Conference, pages 2124-2129.

[8] R. Olfati-Saber. Flocking for multi-agent dynamic systems: Algorithms and theory. IEEE Transactions on Automatic Control, 51(3):401-420, 2006.

[9] B. Paden and S. S. Sastry. A calculus for computing Filippov's differential inclusion with application to the variable structure control of robot manipulators. IEEE Trans. on Circuits and Systems, 34(1):7382, 1987.

[10] D. Shevitz and B. Paden. Lyapunov stability theory of nonsmooth systems. IEEE Trans. on Automatic Control, 49(9):1910-1914, 1994.

[11] H. Tanner and K.J. Kyriakopoulos. Backstepping for nonsmooth systems. Automatica, 39:1259-1265, 2003.

[12] H.G. Tanner, A. Jadbabaie, and G.J. Pappas. Flocking in fixed and switching networks. IEEE Transactions on Automatic Control, 52(5):863-868, 2007. 